\title{
Streamlining protein complex production using multiprotein expression technologies
}

$\underline{\text { Yan Nie }}^{1}$, Fan Zhang ${ }^{1}$, Chao Yuan ${ }^{1}$, Lingyun $\mathrm{Li}^{1}$, Yiling Sun ${ }^{1}$, Yannan Wang ${ }^{1}$, Lixia Zhao ${ }^{1}$, Philip J. Robinson ${ }^{2}$, Imre Berger ${ }^{3}$, Roger D. Kornberg ${ }^{2}$

${ }^{1}$ SIAIS, ShanghaiTech University, Shanghai, China, ${ }^{2}$ Department of Structural Biology, Stanford University School of Medicine, Stanford, United States, ${ }^{3}$ The School of Biochemistry, University of Bristol, Bristol, United Kingdom E-mail: nieyan@shanghaitech.edu.cn

To streamline the recombinant production of multiprotein complexes for basic research and translational medicine, an array of advanced and automatable expression systems have been developed for $\mathrm{E}$. coli, insect and mammalian cells. Those systems, especially the MultiBac system (used in $>1,000$ academic and industrial labs), have greatly contributed to structural and functional studies of numerous important multiprotein complexes.

In this talk, I will present the basic design principles of the MultiBac system and discuss several critical technical aspects when expressing challenging multiprotein complexes in insect cells, as exemplified by our latest results of several essential transcription complexes.

[1] Nie, Y., Chaillet, M., Becke, C., Haffke, M., Pelosse, M., Fitzgerald, D., Collinson, I., Schaffitzel, C. \& Berger, I. (2016). Adv. Exp. Med. Biol., 896, 27-42.

[2] Bieniossek, C., Imasaki, T., Takagi, Y. \& Berger, I. (2012). Trends Biochem. Sci., 37, 49-57.

[3] Trowitzsch, S., Bieniossek, C., Nie, Y., Garzoni, F. \& Berger, I. (2010). J. Struct. Biol., 172, 45-54.

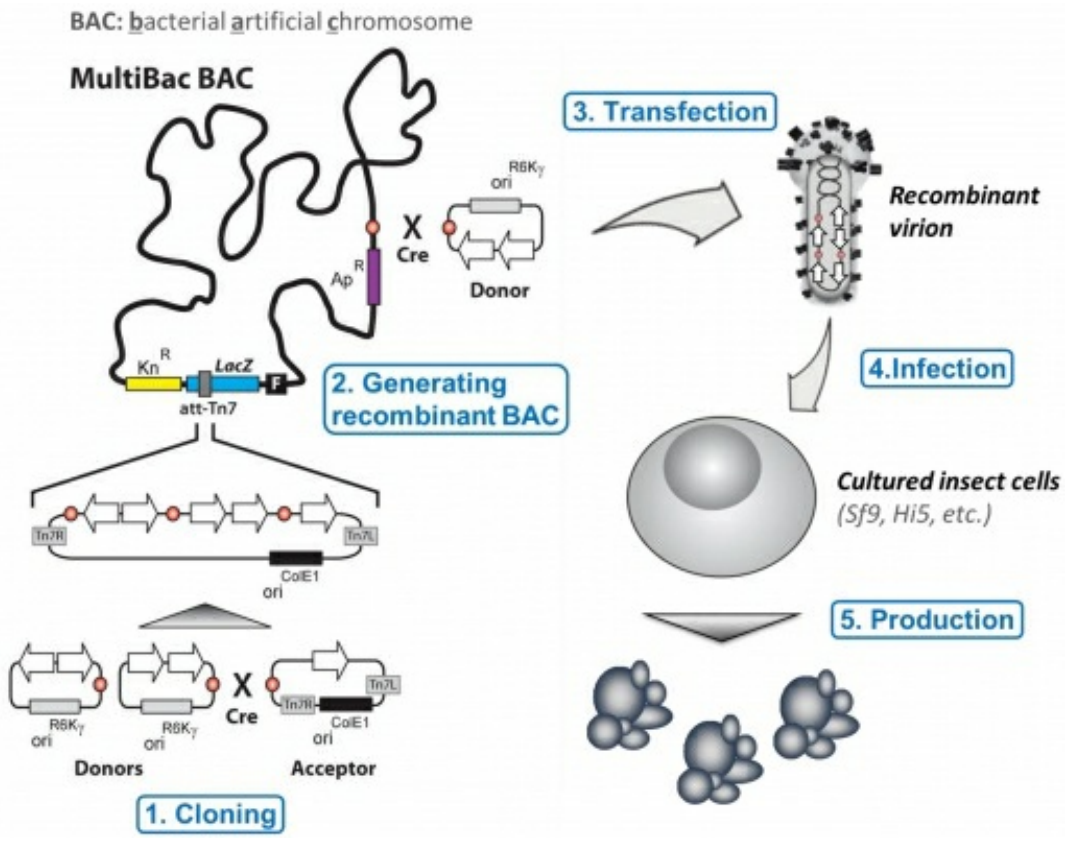

Keywords: Baculovirus Expression Vector System, MultiBac, Transcription Complexes 